


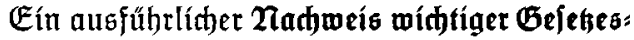
auggaben, ín dem eíne groß̉e 2 năb

\section{Guttentagithen Gammlung}

uno der Gammlungen

Gtílkes Rechtsbíblíothek uno nou ฐ. Grhweíter $\mathfrak{D e t l a g}$

fowíe grőßsere und hleínere Fommentare, Lebr: bürther, Gammelwerke, Entjot)eídungsiamm: Lungen und Beitjthriften verzeíthet find, wird auf $\mathfrak{B u n i t h ~ f i o j t e n l o s ~ z u g e j a n d t . ~}$

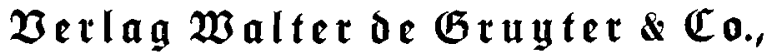
Berlín W 35, Woutrith jtrẩe 13. 


\section{Guttentagitge Sammlung}

Mr. 4

Deutict)er Reiçsgejebe

$\mathfrak{N x} .4$

Rommentare und erläuterte Tertaugaben

\section{Şandelsgejębuch \\ (ohne Seerect)}

Mit Erläuterungen

bot1

\section{Dr. Grnjt Seymann}

Beh. Juftizrat, orb. Frofefior an ber univerfität Berlin

unter Mitarbeit bon

\section{Şano-Wilthelm Rötter}

Ređtşantwalt in Berlin

3roeite, Durdgejebene 2luflage

Det Neubearbeitung

(19. (O) a m tauflage)

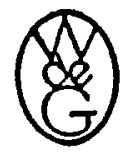

Berrin 1944

$\mathscr{W} \mathfrak{a} \mathfrak{l} \mathfrak{t} \mathfrak{e} \mathfrak{x} \mathfrak{e} \mathfrak{G} \mathfrak{x} \mathfrak{u} \mathfrak{t} \mathfrak{e} \mathfrak{x} \& \mathbb{C} \mathfrak{v}$.

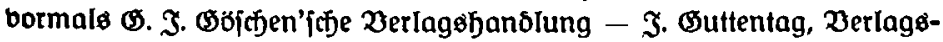
buchbandlung - Georg Reimer - Karl $\mathfrak{T}$. Trübner - Deit \& Comp. 


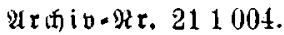

